\title{
Childhood acquired heart disease in Nigeria: an echocardiographic study from three centres
}

Sadoh E. Wilson, ${ }^{1}$ Uzodimma C. Chinyere, ${ }^{2}$ Daniels Queennette. ${ }^{3}$

1. Department of Child Health University of Benin Teaching Hospital, Benin Nigeria

2. Paediatric Department, Lagoon Hospital, Lagos Nigeria

3. Paediatric Department, Zankli Hospital Abuja Nigeria.

\begin{abstract}
Introduction: Acquired heart diseases (AHD) are not uncommon in children. The current multi-center study aims to provide a more representative data of AHD in Nigeria.

Methods: Over 42 months, children referred for echocardiographic evaluation who had confirmed AHD in three centers in Nigeria were recruited. The data was collected on biodata and types of AHD.

Results: There were 116 children with 132 cases of AHD, with a mean age of $6.7 \pm 5.7$ years. The males were 63(54.3\%). Myocarditis/ dilated cardiomyopathy (DCM) 38(28.8\%) was the commonest AHD followed by pericarditis 34 (25.8\%), rheumatic heart disease (RHD) 23 (17.4\%) and hypertrophic cardiomyopathy 18(13.6\%). Endomyocardial fibrosis (EMF) $3(2.3 \%)$ was uncommon.

Conclusion: Nigerian children face a quadruped of AHD namely, myocarditis, DCM, pericardial disease and RHD. As other types of AHD other than RHD are becoming prevalent, there is need to improve the scope of intervention facilities in the country to meet with the demands of the growing afflictions.
\end{abstract}

Keywords: Acquired heart disease; multicentre; Nigeria

DOI: http://dx.doi.org/10.4314/ahs.v14i3:16

\section{Introduction}

Acquired heart diseases (AHD) are diseases affecting the heart and blood vessels that appears in an individual after birth. They are heterogenous group of disorders that arise from damage to the heart and blood vessels by a variety of processes including ischaemic/ hypoxic, metabolic, nutritional, inflammatory and infectious processes. Common AHD include rheumatic fever/ RHD, myocarditis and pericardial diseases. Others include Kawasaki disease and cardiomyopathies.

In Nigeria, AHD contributes between 28.8\% and $68 \%$ of all cardiac anomalies ${ }^{1-3}$. In an earlier study on AHD in Nigeria in $1982^{1}$, RHD, infective pericarditis, endomyocardial fibrosis and dilated cardiomyopathy contributed $35.8 \%, 26.7 \%, 21.7 \%$ and $11.2 \%$ respectively. In later studies ${ }^{2,3}, \mathrm{EMF}$ prevalence had reduced considerably. A recent study conducted in Lagos had revealed that RHD was not the commonest

\section{Corresponding author: \\ Wilson E. Sadoh \\ Department of Child Health, University of Benin Teaching Hospital, PMB 1111, Benin City, Nigeria. \\ E-mail: sadohehi@yahoo.com \\ Phone: +2348028809710}

AHD, having been displaced to the second position by pericarditis.

There are differences in the distribution of AHD globally. While infectious causes such as RHD continue to be a common AHD in developing countries, the contribution of RHD has reduced considerably in developed countries ${ }^{4,5}$. Dilated cardiomyopathy and Kawasaki disease are now the most frequently seen AHD in the western countries where they are responsible for 9 - 19.1/ 100,000 person year ${ }^{6}$ for children 5 years and below and 0.57/100,000 per year ${ }^{7}$ for children under 18 years respectively in the United States. The developing countries continue to contribute the greater share of the 16 million people who suffer from rheumatic fever and RHD, and the 200,000 deaths due to both diseases globally ${ }^{8}$. This high burden of RHD portends grave consequences for the health care delivery of the resource poor countries. The human immune-deficiency virus / acquired immune-deficiency syndrome (HIV/AIDS) also since its advent contributed to the burden of AHD especially in developing countries. Pulmonary hypertension, pericardial disease, left ventricular dysfunction and dilated cardiomyopathy have been described among children with HIV/AIDS ${ }^{10,11}$. It is important to accurately describe the pattern of AHD and their frequencies in the country to enable better health planning and distribution of finances. It will also assist the existing health facilities to channel their resources to cater for those conditions with greater 
impact on the community. A high burden of RHD would emphasize the need for capacity for valvular repair, while high burden of advanced stage of dilated cardiomyopathy and other types of cardiomyopathies would require capacity for ventricular assist devices and heart transplantation.

The advent of echocardiography has afforded better and more accurate diagnosis of cardiac disease, thus accurate prevalence and better description of cardiac diseases that were once suspected or thought to be rare in developing countries are now available. Previous studies on AHD in Nigeria were limited by small numbers in some cases and by being single site studies. A multicentre study would be more representative of the prevailing distribution of AHDs in the country and would also provide a higher number of cases for evaluation. This is the reason for the present multicentre study in Nigeria whose aims are to determine the spectrum of acquired heart diseases among Nigerian children and to identify the age of the children predominantly afflicted with AHD.

\section{Materials and methods}

In this retrospective study, all the children with echocardiographically diagnosed AHD in three Hospitals situated in three large metropolitan cities in Nigeria were recruited. The centers are two large tertiary care private hospitals in Lagos and Abuja respectively and a tertiary government hospital in Benin City. These hospitals serve as referral centers for the neighboring communities and states and thus the subjects were both patients referred from within and outside the centers. The study period was 42 months, from January 2009 to June 2012 in the three centers. Permission was sought and obtained from the respective institutional Ethics Committee, to use the patients' data.

The echocardiography was done by the three pediatric cardiologists in each of the centers. Each child had two Dimensional (2D), M-mode and Doppler examinations in multiple views. The Aloka Prosound SSD-4000SV in Benin and Philips HD7XE were used in Lagos and Abuja. The analysis of the reports was done according to the recommendations of the American Society of echocardiography ${ }^{12}$. The children's age, sex and indication for echocardiography were noted. The types and classification of AHD were also documented using a proforma.

The diagnosis of RHD was based on the 2006 World Health Organisation classification ${ }^{13}$. The type of valvular lesion and the type of RHD, probable, possible or definite RHD were noted. The diagnosis of myocarditis/ dilated cardiomyopathy (DCM) was made on echocardiographic findings of grossly enlarged, hypokinetic heart with poor cardiac function and typical history and findings in the electrocardiogram. The echocardiography was done to define cardiac functions, detect the presence of complications such as pericardial effusion and to exclude other cardiac lesions that could be responsible for the condition. Myocarditis and DCM were grouped together because of the difficulty with telling them apart on echocardiography and the fact that endomyocardial biopsy was not done to confirm cases of myocarditis. Viral studies could not be done to exclude possible viral aetiology, because of non-availability of requisite facility. The diagnosis of pulmonary hypertension was made if the tricuspid valve gradient was $>30 \mathrm{mmHg}$ and the pulmonary valve gradient $>15 \mathrm{mmHg}$ representing pulmonary systolic and diastolic blood pressures. Pericardial effusion (PE) was considered significant when the echo free space between the pericardial linings anywhere around the heart was $>4 \mathrm{~mm}$. The causes of PE were established by doing other ancillary tests. Pericarditis was supported by electrocardiographic findings of ST - segment changes and low voltages. Tuberculous pericarditis was confirmed by positive Acid and Alcohol fast bacilli (AAFB) test from pericardial aspirate. Hypertrophic cardiomyopathy was diagnosed when there was asymmetrical septal hypertrophy and septal dysorganisation on M-mode. When the ratio between the septal and ventricular wall thickness in diastole was $>1.3 \mathrm{~cm}^{14}$, it was also supportive. Infants born to diabetic mothers who had hypertrophic cardiomyopathy were excluded as the condition was considered to be congenital. Right ventricular hypertrophy was diagnosed when the free wall was $>5 \mathrm{~mm}$, measured at end diastole ${ }^{15}$. Other diagnoses were based on standard echocardiographic findings.

The data were coded and entered into SPSS version 16 (Chicago IL) and analysed using the same statistical tool. The frequencies of AHD were presented in simple percentages. Continuous variables were presented in means and standard deviation (SD). The means of continuous variables were compared with student's $t$ test. The association between variables was compared using $\chi^{2}$ test

\section{Results}

There were 116 children recruited for the study, consisting of $63(54.3 \%)$ males and $53(45.7 \%)$ females. 
The mean age was $6.7 \pm 5.7$ years (range; 1 week to 17 ) years. The mean age of the male children $(6.2 \pm 5.9$ years; range- 1 week to 17 years) was lower than that of the females ( $7.4 \pm 5.4$; range-10 days -17 years), but the difference was not statistically significant, $\mathrm{p}=0.19$ $(\mathrm{CI}=-0.67,3.27)$. The ages were categorized into $<5$ years, 5 to $<10$ years, 10 to $<15$ years and $\geq 15$ years with 51 (44.0\%), 20 (17.2\%), $32(27.6 \%)$ and $13(11.2 \%)$ respectively in these categories. Thirty children $(25.9 \%)$ were infants.

Of the 116 children, 16 had double pathologies resulting in 132 cases of AHD. Most of the AHDs were from Benin centre $87(65.9 \%)$ followed by Abuja centre $27(20.5 \%)$ and Lagos centre 18(13.6\%). The distribution of the common cases of AHD was similar in the three centres. Tables I and II shows the distribution of types of AHDs by study centre and gender respectively.

\section{Table I}

The distribution of acquired heart disease cases by study centre

\begin{tabular}{lcccc}
\hline Type of disease & Benin & Study centre & Total (\% \\
& & & Lagos & \\
\hline Myocarditis/ DCM & 30 & 6 & 2 & $38(28.8)$ \\
Pericardial disease & 22 & 4 & 8 & $34(25.8)$ \\
Rheumatic heart disease & 15 & 5 & 3 & $23(17.4)$ \\
Hypertrophic cardiomyopathy & 13 & 4 & 1 & $18(13.6)$ \\
Pulmonary hypertension & 4 & 3 & 2 & $9(6.8)$ \\
Right ventricular hypertrophy & 1 & 3 & 1 & $5(3.8)$ \\
Endomyocardial fibrosis & 2 & 1 & 0 & $3(2.3)$ \\
Infective endocarditis & 0 & 1 & 0 & $1(0.8)$ \\
Coronary aneurysm & 0 & 0 & 1 & $1(0.8)$
\end{tabular}

Total cases

87

27

18

132(100.0)

Of the 132 cases, the commonest AHD was myocarditis / DCM in 38(28.8\%), followed by pericardial disease 34 (25.8\%), RHD 23 (17.4\%) and hypertrophic cardiomyopathies 18 (13.6\%). Pericardial effusion complicated 16 other cases of AHDs. They include $13(81.3 \%)$ cases of myocarditis/ DCM and 3(18.7\%) cases of RHD.

Table I

The distribution of acquired heart disease cases by gender

\begin{tabular}{llll} 
Type of disease & Male & Female & Total (\%) \\
\hline Myocarditis/ DCM & 24 & 14 & $38(28.8)$ \\
Pericardial disease & 22 & 12 & $34(25.8)$ \\
Rheumatic heart disease & 9 & 14 & $23(17.4)$ \\
Hypertrophic cardiomyopathy & 12 & 7 & 18 \\
Pulmonary hypertension & 4 & 5 & $9(6.8)$ \\
Right ventricular hypertrophy & 2 & 3 & $5(3.8)$ \\
Endomyocardial fibrosis & 3 & 0 & $3(2.3)$ \\
Infective endocarditis & 1 & 0 & $1(0.8)$ \\
Coronary aneurysm & 0 & 1 & $1(0.8)$ \\
\hline
\end{tabular}

Pericardial effusion complicated Down syndrome in $8(23.5 \%)$ of which 2 were confirmed to have hypothyroidism. There were $3(8.8 \%)$ cases of tuberculous pericarditis of which one was constrictive pericarditis. PE was present in 3(8.8) cases with HIV associated nephropathy (HIVAN) while the remaining
$4(11.8 \%)$ cases were possibly viral. There were two cases of cardiac tamponade, both with HIVAN, of which one died. The mean age of children with pericardial disease was $5.8 \pm 5.9$ years (range; 3 months to 17 years). Of the 34 cases of $\mathrm{PE}$, congestive cardiac failure was also present in $18(52.9 \%)$. 
Table II

Distribution of acquired heart diseases by age group

\begin{tabular}{lccccc}
\hline Type of disease & $<\mathbf{5 r}$ & $\mathbf{5 - < 1 0 \mathbf { y r }}$ & $\mathbf{1 0 - < 1 5 \mathbf { y r }}$ & $\mathbf{1 5}$ & Total $\mathbf{( \% )}$ \\
\hline Myocarditis/ DCM & 12 & 6 & 7 & 3 & 38 \\
Pericardial disease & 17 & 6 & 6 & 5 & 34 \\
Rheumatic heart disease & 0 & 9 & 10 & 4 & 23 \\
Hypertrophic cardiomyopathy & 6 & 1 & 8 & 3 & 18 \\
Pulmonary hypertension & 6 & 1 & 2 & 0 & 9 \\
Right ventricular hypertrophy & 4 & 0 & 1 & 0 & 5 \\
Endomyoardial fibrosis & 0 & 0 & 2 & 1 & 3 \\
Infective endocarditis & 0 & 1 & 0 & 0 & 1 \\
Coronary aneurysm & 1 & 0 & 0 & 0 & 1 \\
\hline Total $(\%)$ & $45(34.1)$ & $24(18.2)$ & $36(27.3)$ & $16(12.1)$ & $132(100.0)$
\end{tabular}

Figure I shows the short axis parasternal view of a 10 year old male patient showing pericardial effusion around the right ventricle.

The commonest valvular lesion in the RHD was mitral regurgitation (MR) 8(34.8\%). Other cases of valvular lesions were MR and mitral stenosis (MS) combined was 5(21.7\%), MS only was 4(17.4\%), MR and aortic regurgitation (AR) combined was 4(17.4) while MR, MS and AR combined in $2(8.7 \%)$. The mitral valve was thus the most frequently involved valve in all the $23(100.0 \%)$ cases. The mean age of the children with RHD was $10.7 \pm 3.0$ (range; 5 - 15) years.

Figure I. Short axis parasternal view of a 10 year old boy showing pericardial effusion around the right ventricle. Indicated as PE on the image

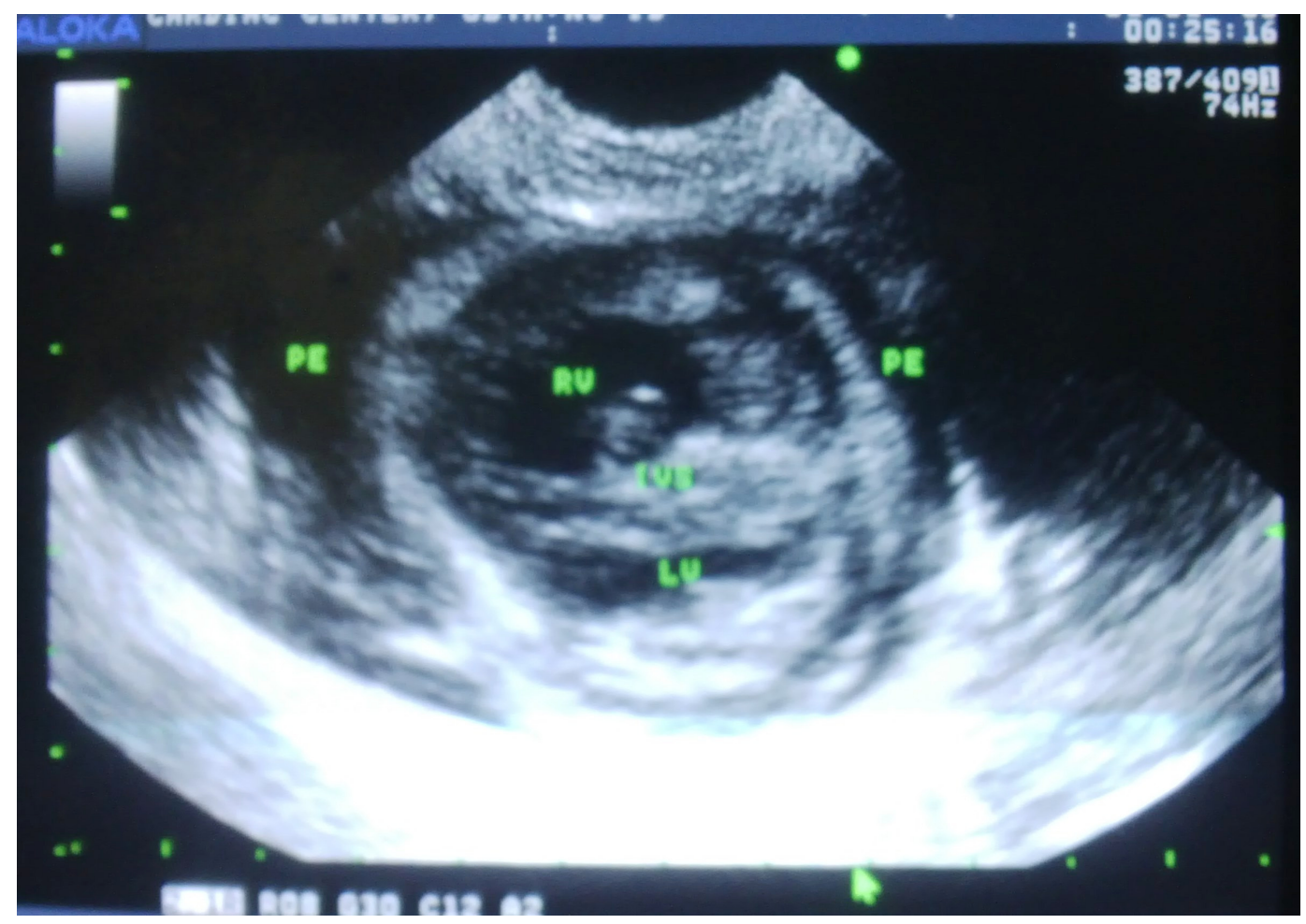

$840 \times 572 \mathrm{~mm}(72 \times 72 \mathrm{DPI})$

Figure II shows the apical two chamber views of a 12 year old female showing thickened mitral valve leaflets in the left panel and significant mitral regurgitation in the right panel.
Hypertrophic cardiomyopathy was seen in $18(13.6 \%)$ cases. It was obstructive in $4(22.2 \%)$ and non-obstructive in $14(77.8 \%)$. Only $2(8.7 \%)$ were concentric, the others were asymmetrical. There were 
$3(16.7 \%)$ cases of proven AIDS stage 4 and one case of an 8 year old with insulin dependent diabetes mellitus. Their mean age was $9.0 \pm 6.4$ years (one week to 17 years). No cause could be adduced to the other cases of hypertrophic cardiomyopathy. Myocarditis/ DCM were present in $38(27.3 \%)$ children with a bimodal age range of 1 week to 3.5 years and 4.5 to 14 years.

Figure II. Apical two chamber view of a 12 year old female showing thickened anterior and posterior mitral valve leaflets on the left panel and severe mitral regurgitation on the right panel

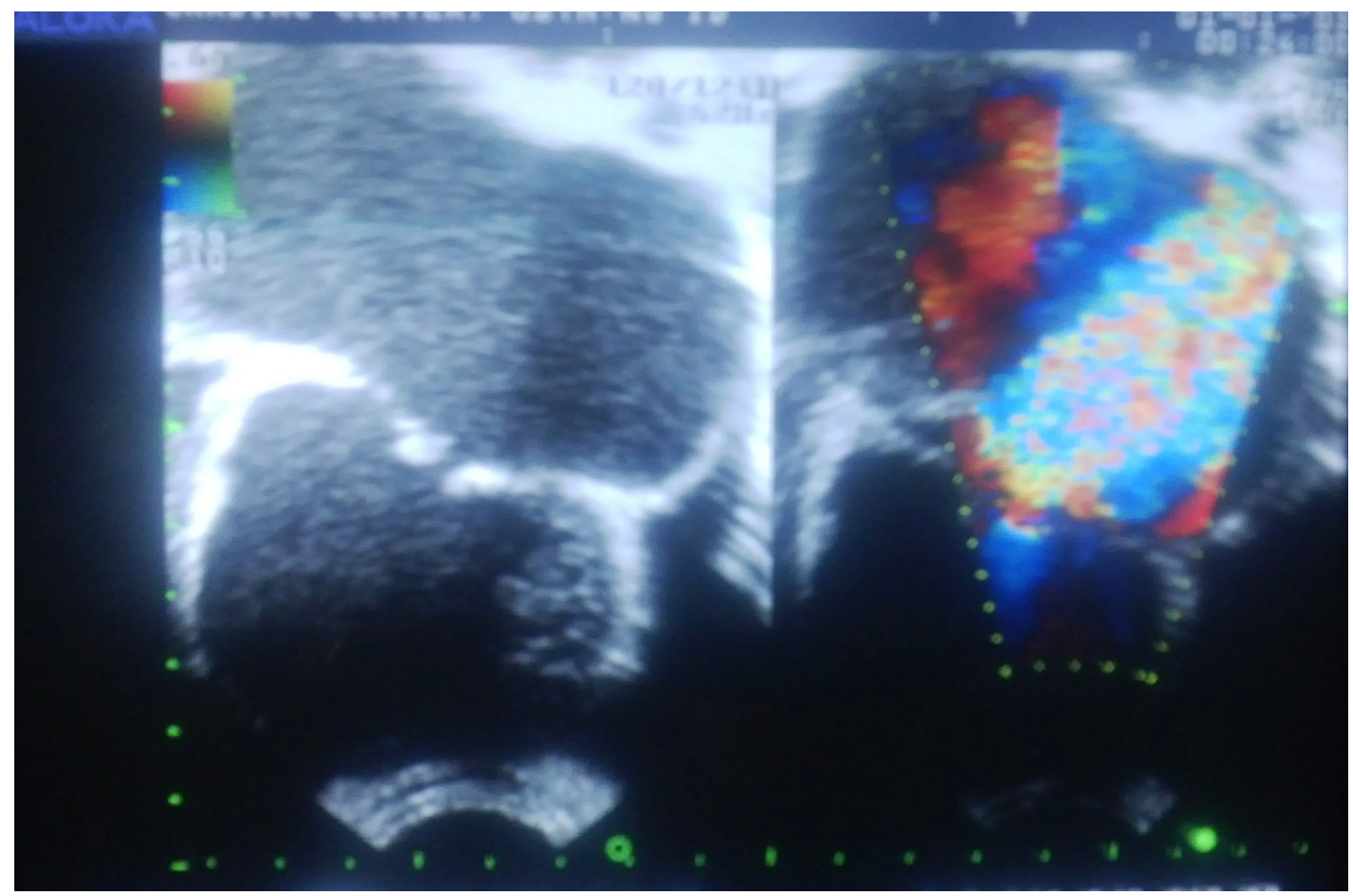

$830 \times 551 \mathrm{~mm}(72 \times 72 \mathrm{DPI})$

There were $32(84.2 \%)$ cases of myocarditis/ DCM presenting in CCF. Of the $6(15.8 \%)$ tested for HIV, 3 were HIV positive. None of the cases of DCM/ myocarditis had hypertension, coarctation of the aorta or a history of anti-cancer chemotherapy. The dilated left ventricular and atrial chambers of a 4 year male child are shown on apical four chamber view in figure III.

Endomyocardial fibrosis (EMF) occurred in $3(2.2 \%)$ of the study population. Their mean age was $12.3 \pm 3.8$ (8 to 15 ) years, two out of these cases were RV type EMF with one case of LV type EMF.
Pulmonary hypertension was found in $9(7.6 \%)$ children. Of these, $6(66.7 \%)$ children had AIDS and recurrent chest infections, while $3(33.3 \%)$ neonates had persistent pulmonary hypertension of the newborn from meconium aspiration syndrome. Five (3.6\%) children with chronic adenoidal hypertrophy on presurgical assessment were found to have right ventricular hypertrophy on echocardiography. They were aged between 1 and 11 years with a mean of $3.7 \pm 4.2$ years. Most of the children were $<5$ years old, see table III. 
Figure III. A pical four chamber view of a four year old boy showing dilated left ventricle and atrium in a child with dilated cardiomyopathy/ myocarditis

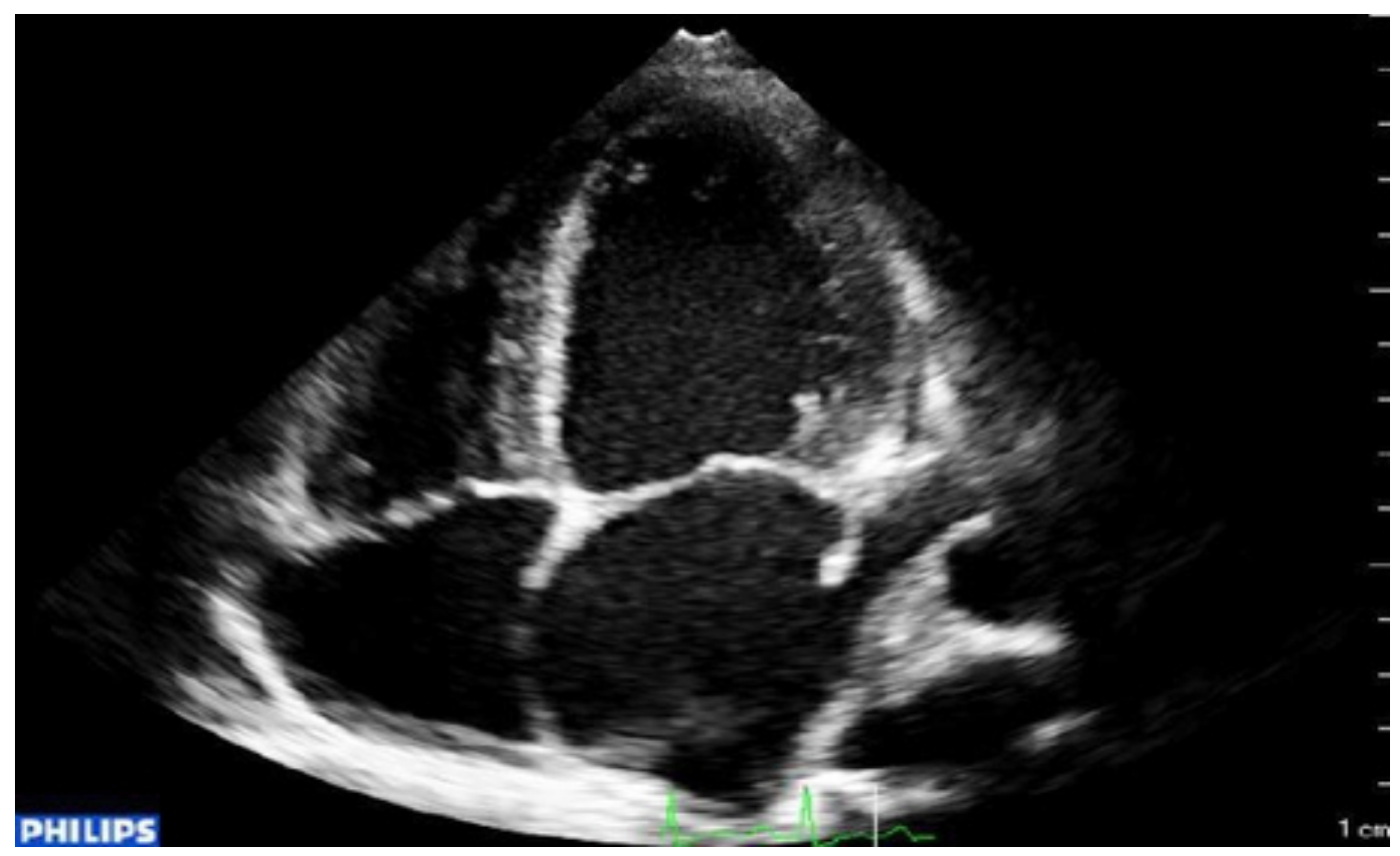

160x112mm (72 x 72 DPI)

\section{Discussion}

The most prevalent AHD in this study was myocarditis/ DCM followed by pericardial disease and then RHD. The finding of RHD not being the commonest AHD is consistent with the Lagos study on structural heart disease in children ${ }^{2}$. These findings may represent a change in epidemiology of AHD in Nigeria. In previous Nigerian studies ${ }^{1,3}$, RHD was the predominant cause of AHDs, being responsible for $68 \%$ of AHD in an echocardiographic study in Jos Nigeria. The proportion of cases with RHD $(17.4 \%)$ in this study is smaller than $68 \%$ obtained among children in Jos Nigeria ${ }^{3}$, the $35.8 \%$ in Ibadan ${ }^{1}$ and the $28.6 \%$ in the Lagos study ${ }^{2}$. It is not clear if the gradual decline in the proportion of RHD amongst AHD in Nigeria represents a true decline in prevalence of RHD. The improvement in housing, environmental sanitation and personal hygiene in the country ${ }^{16}$, the same factors that were identified to reduce the high burden of RF/RHD in the developed countries, could have been responsible for the decline in burden.

Myocarditis and DCM were grouped together because of the difficulty with distinguishing both on echocardiographic evaluation. Both conditions may have evidence of myocardial dysfunction, reduced fractional shortening and ejection fraction, dilated ventricular chambers and segmental wall motion abnormalities ${ }^{17}$. Endomyocardial biopsy, although not a very sensitive test, it is the diagnostic test for myocarditis. The test was however not available in the study centres and thus the cases of myocarditis were suspected. The seeming increasing number of myocarditis and DCM may not be unrelated to the prevalent HIV/AIDS in the country as shown by the finding of $50 \%$ of cases tested for HIV were positive. The increasing contribution of HIV to childhood AHD has been reported by earlier workers $^{10,11}$. A study that evaluates the HIV status of children with suspected myocarditis or DCM would provide the true contribution of HIV to these cases. The availability and utilization of echocardiography in the country may have also contributed to the seeming high numbers of myocarditis and DCM in this study, with patients with unexplained heart failure being referred for echocardiography.

Myocarditis and DCM often presents with congestive cardiac failure, this has similarly been reported in previous studies ${ }^{18,19}$. Myocarditis may herald development of dilated cardiomyopathy. In a study of six cases of DCM in Jos Nigeria ${ }^{19}$, all the children presented with heart failure. It is of note that in that study, all the patients were younger than 5 years, unlike the present study in which the age range was 1.2 to 17 years. The severe cases of dilated cardiomyopathy tend to run a fatal course. The ultimate form of intervention is cardiac transplantation. Dilated cardiomyopathy is a major reason for cardiac transplantation in developed 
countries $^{20}$. The increasing trend of myocarditis/dilated cardiomyopathy suggest the need for pre-emptive expansion of available resources to meet the treatment needs of the affected individuals. It may indicate need for availability of ventricular assist devices as well as heart transplant programs in the country. The lack of and/or affordability of the cost of this form of care is contributing to the childhood morbidity and mortality in Nigeria ${ }^{1,3,19}$.

The young age of some of the subjects in this study reflects the early and prompt investigation of children with possible cardiovascular disease and buttresses the importance of the presence of cardiac centres with diagnostic facilities in improving access to care. The prevalence of hypertrophic cardiomyopathy in this study is high and is consistent with finding in the Baltimore-Washington Infant study ${ }^{21}$ in which hypertrophic cardiomyopahty was the commonest cardiomyopathy documented.

Pericardial disease which was the second commonest AHD in this study, in addition to other underlying cardiac and non-cardiac conditions was associated with congestive cardiac failure in most of the cases. Cardiac failure and PE complicating AHD in some of these children is consistent with a previous study $^{22}$. In a previous study in Nigeria ${ }^{23}$, mycobacterium tuberculosis and staphylococcus aureus were the commonest causative agent of pericarditis. In this study the true prevalence of tuberculous pericarditis could not be determined since it was only evaluated for 11 children. The usefulness of echocardiography is shown in aiding the aspiration of pericardial fluid to ease the tamponade effect on the heart in one case of cardiac tamponade. The other cases of cardiac tamponade died before intervention was instituted. Echocardiography also facilitated the aspiration of the effusion of three cases. This further shows its interventional role in clinical practice.

The age range of children with RHD in this study is similar to values obtained from previous studies in children. Also in previous studies ${ }^{1-3}$, mitral valve disease was the commonest valvular involvement in the cases of RHD. The pulmonary valve was not involved in our patients and this agrees with previous studies ${ }^{3}$.

Endomyocardial fibrosis that was quite prevalent in Nigeria in the time past ${ }^{1}$, especially in the Southern part of the country, seems to be on the decline. The low number recorded in this study is consistent with more recent reports in the country ${ }^{2,24}$. Only one case of Kawasaki's disease with coronary arterial abnormality was recorded in this study. Kawasaki disease is one of the leading causes of AHD in developed countries. It is not clear if the small number of Kawasaki disease in this study represents a true low prevalence or underestimation of the true prevalence due to wrong clinical diagnosis and consequently, failure to refer affected patients for cardiovascular evaluation.

Pulmonary hypertension is a major cardiovascular finding that has been documented in children with AIDS by other workers ${ }^{10,11,25}$. The long term survival of children with HIV/AIDS because of the availability of anti-retroviral medicines, may mean the health care system will see increasing numbers of such cardiovascular complications. The availability of echocardiography facility and its utilization for such indication as pre-surgical evaluation of children has contributed to the identification of these conditions as has been found in studies in developed countries ${ }^{27}$. It is been shown that the cardiac hypertrophy and other cardiopulmonary features of adenoidal hypertrophy regresses following treatment ${ }^{26}$.

In this study, we acknowledge some limitations. Endomyocardial biopsy was not done for suspected cases of myocarditis/ myocarditis thus the cases could not be confirmed. The HIV statuses of suspected cases of myocarditis/ DCM were not determined for most cases as some of the cases were referred from facilities outside the study centers. Thus the true contribution of HIV to causation of myocarditis/ DCM could not be ascertained. Genetic studies could not be done for the cases of DCM and HCM, this is important as some of these cases may have been inherited and thus would not strictly be AHD. This multi-center study was conducted in referral centers, thus the reported prevalences may not represent the true prevalences in Nigeria. Perhaps a clearer picture may be seen when the large multicentered study involving all six geopolitical zones of Nigeria is concluded.

\section{conclusion}

The current spectrum of AHD in this study is changing from previous pattern reported earlier from the country. Most of the children were young availing the opportunity to diagnose their conditions early and institute early intervention. Interestingly too, myocarditis/ DCM and pericardial diseases are more prevalent than RHD that used to dominate the spectrum of AHD in Nigeria. The proportion of RHD and EMF seem on the decline, although RHD was not the leading cause of AHD in this study, it is still a major cause of morbidity in Nigeria. The number of children with myocarditis and Dilated cardiomyopathy 
is increasing. There is need to re-direct attention to improved diagnostic acumen for myocarditis by increasing awareness, alongside improved efforts to provide interventional facilities to cater for the growing affliction.

\section{References}

1. Jaiyesimi F. Acquired heart disease in Nigerian children: an illustration of the influence of socioeconomic factors on disease pattern. J Trop Pediatr 1982; 28: $223-9$

2. Okoromah CAN, Ekure EN, Ojo OO, Animashaun $\mathrm{BA}$, Bastos MI. structural heart disease in children: profile, problems and prospects. Niger Postgrad Med J 2008; 15: $82-88$

3. Bode-Thomas F, Okolo SN, Ekedigwe JE, Kwache IY, Adewunmi O. Paediatric echocardiography in Jos University Teaching Hospital: problems, prospects and preliminary audit. Nig J Paediatr 2003; 30: 143 - 149

4. Gordis L. The virtual disappearance of rheumatic fever in United States: lesson in the rise and fall of the disease. T Duckett Jones memorial lecture. Circulation 1985; $72: 1155-62$

5. World Health Organisation. 2001. Rheumatic fever and rheumatic heart disease. Report of a WHO Study Group. Geneva WHO Tech report Ser. 2001. 923. http://www.who.int/cardiovascular_diseases/ resources/trs923/en/ (accessed August 2011)

6. Towbin JA, Lowe AM, Colan SD, Sleeper LA, Orav EJ, Clunie $S$ et al. Incidence, causes and outcomes of dilated cardiomyopathy in children. JAMA 2006; 296: $1867-76$

7. Belay ED, Holman RC, Clarke MJ, Destefano F, Shahriari A, Davis RL et al. The incidence of Kawasaki syndrome in West Coast Health maintenance organizations. Pediatr Infect Dis J 2000; 19: 823 - 32

8. Karthikeyan G, Mayosi BM. Is primary prevention of rheumatic fever the missing link in the control of rheumatic heart disease in Africa? Circulation 2009; 120: $709-713$

9. Ekra A, Bertrand E. Rheumatic heart disease in Africa. African Health 1994; 16: 32 - 3

10. Okoromah CAN, Ojo OO, Ogunkunle OO. Cardiovascular dysfunction in HIV-infected children in a sub-Saharan African country: comparative cross sectional observational study. J Trop Pediatr 2011; Feb [Epub ahead of print]

11. Rajeshwari K, Amiritsinh SP, Mandal RN, Kurian S, Anuradhi S. Cardiac abnormalities in HIV infected children presenting to a tertiary level teaching hospital at New Delhi. Brit J Med Med Res 2014; 4: 237 - 43
12. Amerian Society of Echocardiography.

Recommendations for continous quality improvement in echocardiography. J Am Soc Echocardiogr 1995; 8: S1 $-28$

13. Carapetis JR, Paar J, cherian T. Standardization of epidemiologic protocols for surveillance of post-streptococcal sequalae: acute rheumatic fever, rheumatic heart disease and acute post-streptococcal glomerulonephritis. WHO Technical Report 2006.

14. Snider AR, Serwer GA, Ritter SB. Echocardiography on pediatric heart disease. $2^{\text {nd }}$ ed. St. Louis: Mosby Publishing. 1997. P. 515 - 22

15. Baker BJ, Scovil JA, Kane JJ, Murphy ML. Echocardiographic detected of right ventricular hypertrophy. Am Heart J 1983; 105: 611 - 14

16. Ebong RD. Environmental health knowledge and practice survey among secondary school children in Zaria, Nigeria. Environ Hlth Perspect 1994; 102: 310 $-2$

17. Pinamonti B, Alberti E, Cigalotto A, Dreas L, Salvi A, Silvestri F, Camerini F. Echocardiographic findings in myocarditis. Am J Cardiol 1988; 62: 285 - 291

18. Guglin M, Nallamshetty L. Myocarditis: diagnosis and treatment. Curr Treat Option Cardiasc Med 2012: Aug 28 [Epub ahead of print].

19. Bode-Thomas F, Ebonyi AO, Animashaun BA. Childhood dilated cardiomyopathy in Jos, Ngeria. Sahel Med J 2005; 8: 100 - 5

20. Singh TP, Almond CS, Piercey G, Gauvreau K, Current outcomes in US children with cardiomyopathy listed for transplantation. Cir Heart Fail 2012; 5: 594 60

21. Ferencz C, Neil CA. Cardiomyopathy in infancy observations in a epidemiologic study. Pediatr Cardiol 1992; 13: $65-71$

22. Kessler KM, Rodriguez D, Rahim A, Dheen M, Samet P. Echocardiographic observations regarding pericardial effusions associated with cardiac disease. Chest 1980; 78: 736 - 40

23. Jaiyesimi F, Abioye AA, Antia AU. Infective pericarditis in Nigerian children. Arch Dis Child 1979; 54: $384-90$

24.IkeSO,OnwubereBJC,AnisiubaBC.Endomyocardial fibrosis; decreasing prevalence or missed diagnosis? Niger J Clin Pract. 2003; 6: 95 - 8

25. Rerkpattanapipat $\mathrm{P}$, Wongpraparut N, Jacobs LE, Kotler MN. Cardiac manifestations of acquired immunodeficiency syndrome. Arch Intern Med 2000; 160: $602-8$

26. Abdel-Aziz M. Asymptomatic cardiopulmonary changes caused by adenoidal hypertrophy. J Craniofac Surg 2011; 22: 1401 - 3 